Research Article

\title{
The Meridian Tropism and Classification of Red Yeast Rice Investigated by Monitoring Dermal Electrical Potential
}

\author{
Meng-Tian Wang, ${ }^{1}$ Qiao-Juan He, ${ }^{2}$ Jing-Ke Guo $\left(\mathbb{D},{ }^{3}\right.$ Shu-Tao Liu $\left(\mathbb{D},{ }^{1} \mathrm{Li} \mathrm{Ni},{ }^{4}\right.$ Ping-Fan Rao, ${ }^{2}$ \\ Tian-Bao Chen, ${ }^{5}$ Sheng-Bin Wu, ${ }^{3}$ Shuai-Jun Zhao, ${ }^{3}$ Jia-Hui Qiao, ${ }^{3}$ Peng-Wei Zhang, \\ and Yu-Bo Li ${ }^{7}{ }^{7}$ \\ ${ }^{1}$ Institute of Biotechnology, Fuzhou University, Fuzhou 350002, Fujian, China \\ ${ }^{2}$ School of Food Science and Biotechnology, Zhejiang Gongshang University, Hangzhou 310035, Zhejiang, China \\ ${ }^{3}$ Department of Food and Biological Engineering, Zhicheng College, Fuzhou University, Fuzhou 350002, Fujian, China \\ ${ }^{4}$ Institute of Food Science and Technology, Fuzhou University, Fuzhou 350108, Fujian, China \\ ${ }^{5}$ Natural Drug Discovery Group, School of Pharmacy, Queen's University Belfast, Belfast BT9 7BL, Northern Ireland, UK \\ ${ }^{6}$ Center for Preventive Treatment and Health Management, The Affiliated Hospital of Hangzhou Normal University, \\ Hangzhou 310015, Zhejiang, China \\ ${ }^{7}$ College of Information Science and Electronic Engineering, Zhejiang University, Hangzhou 310027, Zhejiang, China
}

Correspondence should be addressed to Jing-Ke Guo; 2010021@qq.com and Shu-Tao Liu; stliu@fzu.edu.cn

Received 21 May 2021; Revised 20 July 2021; Accepted 14 August 2021; Published 21 August 2021

Academic Editor: Li Zhang

Copyright (c) 2021 Meng-Tian Wang et al. This is an open access article distributed under the Creative Commons Attribution License, which permits unrestricted use, distribution, and reproduction in any medium, provided the original work is properly cited.

Red yeast rice is a traditional Chinese medicine and food that has been purported to color food, ferment, and lower cholesterol. In order to study the antioxidative capacity of red yeast rice and the effects on electrical potential difference (EPD) of 12 acupuncture meridians, the $\mathrm{pH}$ value, oxidation reduction potential (ORP), ABTS, FRAP, T-SOD, and particle size distribution of red yeast rice were analyzed. 20 volunteers were recruited and randomly divided into two groups, the red yeast rice group (10 g red yeast rice and $40 \mathrm{~g}$ water) and control CK group (50 g water). The left 12 acupuncture meridians' EPD was real-time monitored. Samples were taken at the 10th minutes. The whole procedure continued for 70 minutes. It is shown that the $\mathrm{pH}$ value of the red yeast rice was 4.22, the ORP was $359.63 \mathrm{mV}$, the ABTS was $0.48 \mathrm{mmol}$ Trolox, the FRAP was $0.08 \mathrm{mmol} \mathrm{FeSO}_{4}$, the T-SOD was $4.71 \mathrm{U}$, and the average particle size was $108 \mathrm{~nm}(7.1 \%)$ and $398.1 \mathrm{~nm}$ (92.9\%). The results of 12 acupuncture meridians' EPD showed that the red yeast rice can significantly affect the EPD of stomach, heart, small intestine, and liver meridians.

\section{Introduction}

As traditional Chinese medicinal material with dual functions of medicine and food, red yeast rice has been widely applied in food coloring, fermentation, cholesterol reduction, and other aspects [1]. In addition, the red yeast rice contains many bioactive substances, including multiple ketone isoflavones, plant sterols, and unsaturated fatty acids and [2]. The effect of red yeast rice cholesterol mainly comes from the monacolin $\mathrm{K}$ composition. It has a same kind of structure of the compound with lovastatin and contains antiinflammatory and antioxidant activity of the material, such as sterol, dimer acid, and tannin [3].
Acupuncture meridians can be used as a reference index to observe the physiological effects of acupoint intervention and functional substances that can be used as medication and food $[4,5]$. In traditional Chinese theories, meridian tropism refers to the selective therapeutic effects of a medication and food on a certain part of the body. A medication and food may elicit evident or specific therapeutic action on the pathological changes in one or several acupuncture meridians. Meridian tropism theory is most important because it has guided clinical practice for thousands of years in Eastern Asia [6]. In recent years, the explanation of meridian tropism theory by modern scientific techniques would facilitate the understanding and application of traditional Chinese medicine. The urinary 
excretion rate, loss in weight of rats, and the electrolyte levels in the plasma were measured in order to study the meridian tropism for Tinglizi (Semen Lepidii Apetali), Yiyiren (Semen Coicis), and Cheqianzi (Semen Plantaginis) [7]. The relationship between the modified Wuzi Yanzong prescription and meridian tropism was studied according to the tissue distributions of the metabolites, mainly flavanoid compounds in rats [8]. The distribution of tryptanthrin in rat tissues, following oral administration at a dose of $100 \mathrm{mg} / \mathrm{kg}$, was studied in order to characterize the relationship with meridian tropism of indigo naturalis [9]. The dermal acupuncture meridian electrical potential difference (EPD) also can be real-time monitored to observe and compare various medications' and foods' meridian tropism, for volunteers' acupoint antioxidant intervention $[10,11]$ and taking medicine and food functional materials, such as Cordyceps militaris [12], Inonotus obliquus (Fr.) Pilat [13], Dendrobium officinale Kimura et Migo [14], herbal drink $[15]$, tea $[16,17]$, and so on.

Therefore, in order to study the antioxidative capacity of red yeast rice and the effects on EPD of 12 acupuncture meridians (the meridian tropism of red yeast rice), the $\mathrm{pH}$ value, oxidation reduction potential (ORP), ABTS, FRAP, T-SOD, and particle size distribution of red yeast rice were analyzed and the left 12 acupuncture meridians EPD were real-time monitored. This study mainly conducted physical and chemical analysis of red yeast rice in vitro and found that the EPD of 12 acupuncture meridians changed differently after volunteers took red yeast rice to provide a new idea for the application and development of red yeast rice.

\section{Materials and Methods}

2.1. Materials. Red yeast rice (Fujian screen red Bio Technology Co., Ltd., China); the total antioxidant capacity detection kit (ABTS method) (Shanghai beyotime Biotechnology Co., Ltd., China, Batch No. 092518190324); the total antioxidant capacity detection kit (FRAP method) (Shanghai beyotime Biotechnology Co., Ltd., China, Batch No. 1113118190524); and superoxide dismutase (T-SOD) assay kits (Nanjing Jiancheng Bioengineering Institute, China, Batch No. 20190511) were used.

A CS-700 Y crusher (Wuyi Haina Electric Appliance Co., Ltd., China); pH meter (American EUTECH company, US); ORP (Oxidation Reduction Potential) Analyzers (ORP electrode InLab Redox, Mettler Toledo Instrument Shanghai Co., Ltd., China); CF 15 RX II high-speed centrifuge (HITACHI, Japan); Zetasizer Nano laser particle size analyzer (Malvern Instrument Co., Ltd., UK); NH-4 digital display electronic constant temperature water bath (Jiangsu Guohua Electric Appliance Co., Ltd., China); KQ $5200 \mathrm{E}$ type ultrasonic cleaner (Kunshan Ultrasonic Instrument Co., Ltd., China); UV-1700 UV spectrophotometer (Tsujima Corporation, Japan); FlexStation 3 Microplate Reader (Molecular Devices, Inc., US); Ag/AgCl disposable electrocardiogram (ECG) electrode (Shanghai Junkang Medical Equipment Co., Ltd., China); and RM 6240C Multi-channel Physiological Signal Acquisition and Processing System (Chengdu Instrument Factory, China) were used.
2.2. Human Subjects. This study was performed with central enrollment and allocation by the SIBS.CAS-Zhejiang Gongshang University Joint Centre for Food and Nutrition Research (research center). 20 healthy volunteers who met the inclusion criteria were recruited and screened as experimental subjects from October 2018 to July 2019. All volunteers signed the informed consent voluntarily under the condition that they could fully accept the experimental scheme. This experiment was approved by the Ethics Committee of the SIBS.CAS-Zhejiang Gongshang University Joint Centre for Food and Nutrition Research (31500685-3).

(i) Inclusion criteria: (1) age: $>18$ years, regardless of gender; (2) voluntarily signing the informed consent; (3) not taking breakfast before the test; and (4) keeping regular diet and rest.

(ii) Exclusion criteria: (1) having kidney, liver, and cardiovascular disease or other serious diseases; (2) having bad habits, such as smoking, drinking, and drug abuse; (3) long-term drug treatment; (4) taking breakfast before the test; and (5) pregnant women and those with hyperactivity of fire due to yin deficiency.

2.3. Randomization. A computer-generated random number was assigned to each participant. Volunteers were randomly divided into two groups: the red yeast rice group (10 g red yeast rice and $40 \mathrm{~g}$ water) and the control CK group (50 $\mathrm{g}$ water), with the ratio of $1: 1$. The investigator had no clinical involvement in the trial.

2.4. Treatment Protocol. After the recruitment of volunteers was completed, it was uniformly stipulated that they should complete the monitoring in the morning from $8: 30$ am to $10: 30 \mathrm{am}$. The changes of EPD were observed before and after the volunteers took the sample. This was repeated three times, with each time interval of more than 7 days. The volunteers were conscious, placed in a supine position, and asked to breathe calmly. The acupoints were localized according to World Health Organization standard acupoint locations in the Western Pacific Region [18]. The Source-Sea (Yuan-He) acupoint pairs of the 12 meridians on the left were selected as the measurement points of the meridian EPD, with the Sea acupoints (proximal) as the positive pole and the Source acupoints (distal) as the negative pole, respectively (Table 1) [10]. The hair on the selected detection acupoints was trimmed. After previously disinfected with medical alcohol, the acupoints were connected to a digital potentiometer via $\mathrm{Ag} / \mathrm{AgCl}$ disposable ECG electrodes and the EPD of 12 meridians of volunteers was monitored in real time. When monitoring to 10 minutes, the prepared red yeast rice or water should be taken, and it should be taken within $5 \mathrm{~min}$. A total of $70 \mathrm{~min}$ was monitored before and after the administration.

2.5. Sample Preparation. Weighing about $18 \mathrm{~g}$ of red yeast rice and grinding it several times with a grinder, the red yeast 
TABLE 1: Acupuncture point selection of acupuncture meridians.

\begin{tabular}{lcc}
\hline Meridian & Sea (He) acupoints & $\begin{array}{c}\text { Source (Yuan) } \\
\text { acupoints }\end{array}$ \\
\hline $\begin{array}{l}\text { Lung meridian } \\
\text { Large intestine meridian }\end{array}$ & Chize LU5 & Taiyuan LU9 \\
Stomach meridian & Zusanli ST36 & Hegu LI14 \\
Spleen meridian & Yinlingquan SP9 & Taibai SP3 \\
Heart meridian & Shaohai HT3 & Shenmen HT7 \\
Small intestine meridian & Xiaohai SI8 & Wangu SI4 \\
Bladder meridian & Weizhong BL40 & Jinggu BL64 \\
Kidney meridian & Yingu KI10 & Taixi KI3 \\
Pericardium meridian & Quze PC3 & Daling PC7 \\
Triple energizer & Tianjing TE10 & Yangchi TE4 \\
meridian & Yanglingquan & Qiuxu GB40 \\
Gallbladder meridian & GB34 & Taichong LR3 \\
\hline Liver meridian & Ququan LR8 &
\end{tabular}

rice powder was obtained. After 60 mesh sieves, $10 \mathrm{~g}$ of it was accurately weighed and dissolved in $40 \mathrm{~g}$ of distilled water. The mixture was ultrasonized for $20 \mathrm{~min}$ and extracted by using a water bath thermostatic agitator for 1 hour at $37^{\circ} \mathrm{C}$ and $700 \mathrm{r} / \mathrm{min}$ and centrifuged at $25^{\circ} \mathrm{C}$ for $5000 \mathrm{r} / \mathrm{min}$ for $15 \mathrm{~min}$; the supernatant was taken as the sample to be tested and stored in a refrigerator at $-20^{\circ} \mathrm{C}$ for later use.

2.6. Determination of $p H$ Value. The sample should be calibrated with a standard solution before testing. After calibration, the electrode is completely passed over the sample to be tested. When the data are stable, the reading is recorded, and the measurement is repeated three times to take the average value.

2.7. Determination of REDOX Potential (ORP). An appropriate amount of the sample was placed in a beaker for determination. The sample was immersed in the electrode, and the determination was repeated three times to take the average value and the determination time should not exceed 3 min.

2.8. Antioxidant Activity Determination. The total antioxidant capacity (ABTS and FRAP) and SOD activity of samples were analyzed with colorimetric assay kits (Shanghai beyotime Biotechnology Co., Ltd., China, and Nanjing Jiancheng Bioengineering Institute, China).

2.9. Particle Size Distribution Determination. Red yeast rice solution was detected using a Zetasizer Nano particle size tester. $1 \mathrm{~mL}$ sample was taken and placed in the detection dish. The relevant parameters of the instrument were set as temperature $25^{\circ} \mathrm{C}$, scattering angle $173^{\circ}$, and preheating time $2 \mathrm{~min}$, and each cycle was scanned 3 times.

2.10. Statistical Analysis. The study design called for at least 20 healthy subjects. Sample size estimations were not performed. Because of the complex design of this pilot study, sample size was chosen on the basis of practical considerations. Therefore, this study was not designed to have sufficient power, and the results of statistical testing have to be interpreted as descriptive, explorative, and hypothesis generating rather than as confirmatory. Data are reported as means \pm standard deviation. The statistical analysis was performed with IBM SPSS Statistics 22. Results in patients and healthy subjects were compared using the two-sample t-test.

\section{Results}

3.1. Influence of Red Yeast Rice on Meridian EPD. We screened 20 healthy volunteers into the study. All of them completed the study. The study flow is presented in Figure 1.

In this study, after volunteers took red yeast rice or water, the EPD of 12 meridians on the left side of volunteers was monitored in real time for $70 \mathrm{~min}$. The results are shown in Figures 2-4. The EPD changes of the control CK group are relatively gentle, and the fluctuation range is within $10 \mathrm{mV}$. Red yeast rice had obvious effects on the EPD of stomach, heart, small intestine, and liver meridians. After the administration of red yeast rice, the fluctuation range of the meridian EPD of the volunteers exceeded $10 \mathrm{mV}$, the peaks of the stomach, small intestine, and liver meridians all appeared at 40-50 $\mathrm{min}$, and the peaks of the heart meridians appeared at 50-70 min, as shown in Figure 1. For the lung meridian and pericardium meridian, the fluctuation range of the meridian EPD of the volunteers was about $10 \mathrm{mV}$ (Figure 2).

The EPD fluctuation of the meridians of the large intestine, spleen, bladder, kidney, triple energizer, and gallbladder of volunteers in the red yeast rice group is not different from that in the control CK group, as shown in Figure 3 .

3.2. Antioxidant Activity of Red Yeast Rice and Distilled Water. The $\mathrm{pH}$ value, REDOX potential value (ORP), ABTS, FRAP, and T-SOD of red yeast rice and distilled water were determined as shown in Table 2. Distilled water, as a solvent, has a certain REDOX potential value [19]. When the REDOX potential value of red yeast rice is lower than that of distilled water, it indicates that red yeast rice presents reductivity.

3.3. Particle Size Distribution of Red Yeast Rice. The average particle size of red yeast rice soup was $108 \mathrm{~nm}(7.1 \%)$ and $398.1 \mathrm{~nm}$ (92.9\%), respectively.

\section{Discussion}

In our studies of reactive oxygen species (ROS) distribution in living animals by ROS indicator visualization, a few vertical fluorescent lines were demonstrated on the body. They could be almost perfectly superimposed on a standard human acupuncture meridian network [20, 21]. ROS has a strong connection with REDOX potential (ORP) and antioxidant activity. Moreover, topical application of antioxidants to acupoints was found to result in an 


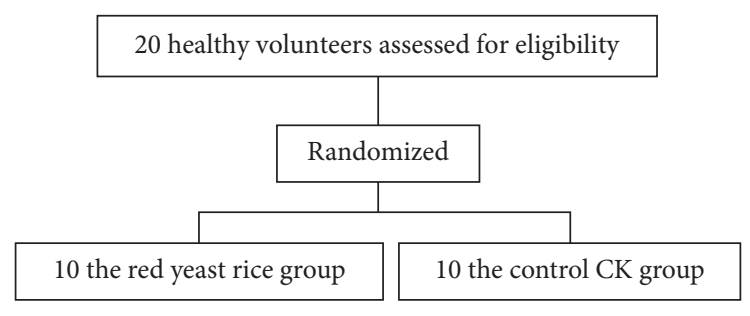

Figure 1: Study flow chart.

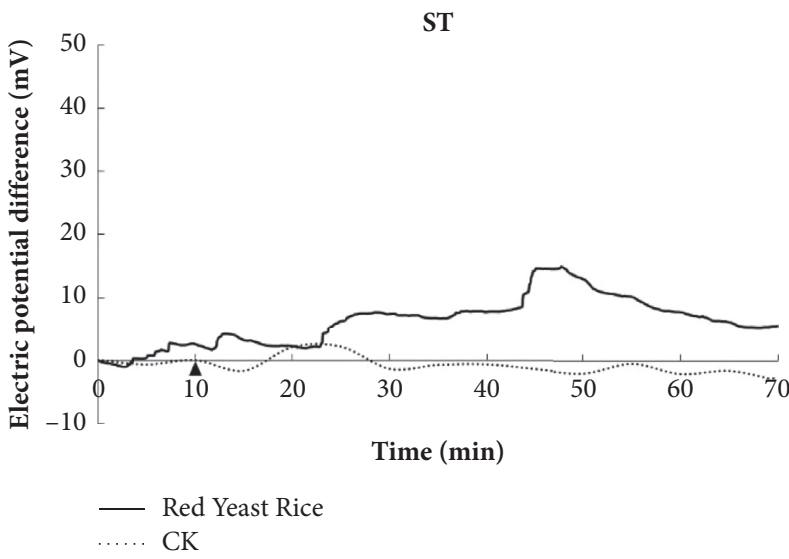

(a)

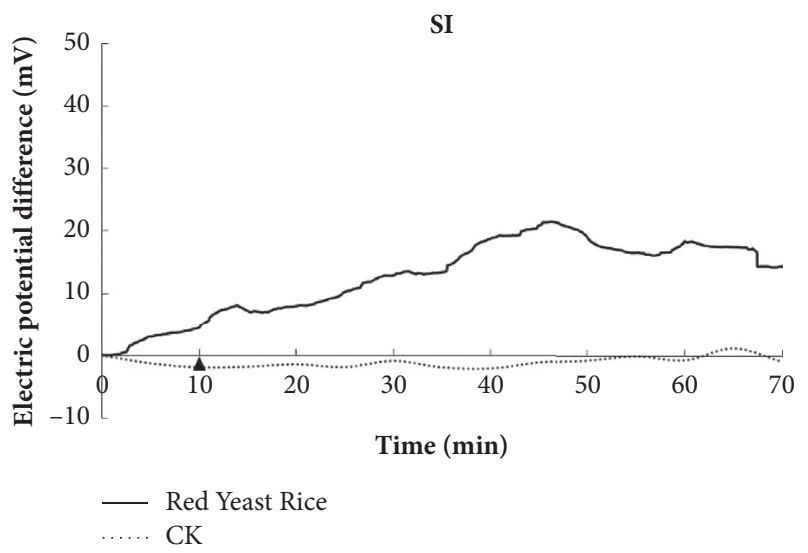

(c)

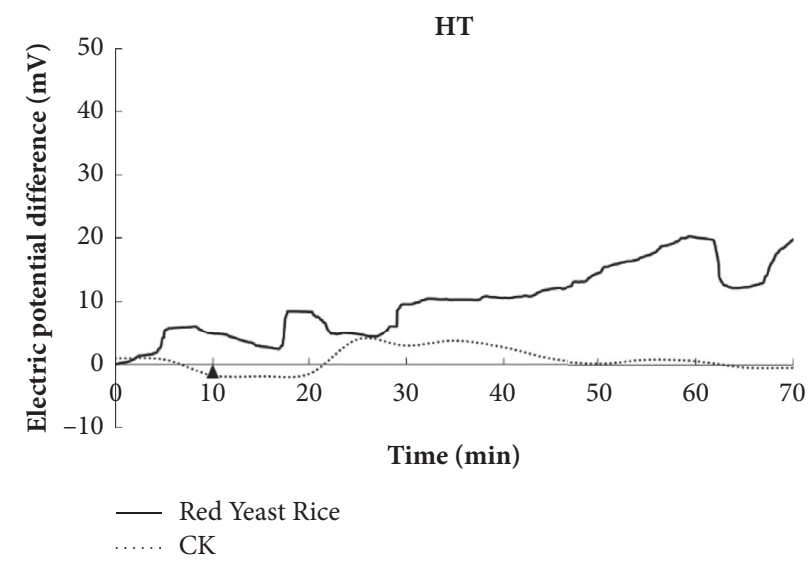

(b)

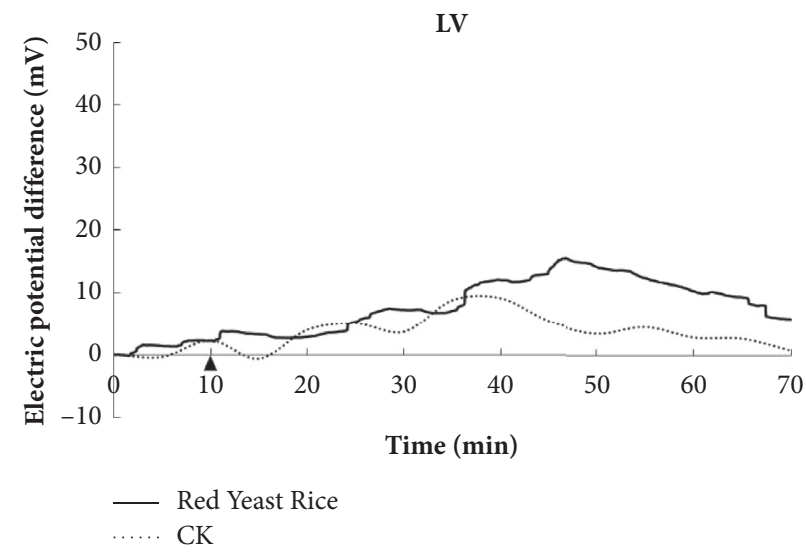

(d)

FIGURE 2: Electric potential difference of the stomach meridian (a), heart meridian (b), small intestine meridian (c), and liver meridian (d).

acupuncture-like action $[10,11,22,23]$. All those results point to a new applaudable hypothesis of meridians as channels of ROS and ROS modulation along meridians as the acupuncture mechanism.

Although in Table 2, the results of antioxidant activity of red yeast rice in vitro show weak acidity $(\mathrm{pH}=4.22 \pm 0.19$, high $\mathrm{H}^{+}$solubility, and partial oxidation), its overall REDOX potential was lower than that of distilled water and showed high reductivity. The results of ABTS, FRAP, and T-SOD all showed that red yeast rice had antioxidant activity. The particle size distribution of red yeast rice soup was determined. The particle size distribution was mainly divided into two components. The main component was $398.1 \mathrm{~nm}$ (92.9\%), followed by $108 \mathrm{~nm}$ (7.1\%). The active ingredients isolated from Semen Armeniacae Amarum with particle size less than $200 \mathrm{~nm}$ had strong biological activity [24]. In addition, enzymatically active nanoparticles of $\mathrm{Cu} / \mathrm{Zn} \mathrm{SOD}$ were simultaneously generated during the reaction, with an average particle size of $175.86 \pm 0.71 \mathrm{~nm}$ [25]. The nanoparticles isolated from sundried Isatis indigotica Fort. root decoction were also about $120 \mathrm{~nm}$ [26]. The nanoparticles less than $200 \mathrm{~nm}$ can enter the macrophages, significantly reduce the oxidative stress level of cells, and show the strong oxidation resistance [27], with the effect of detoxification [28].

When substances with antioxidant activity are introduced into the human body, they will cause changes in the 


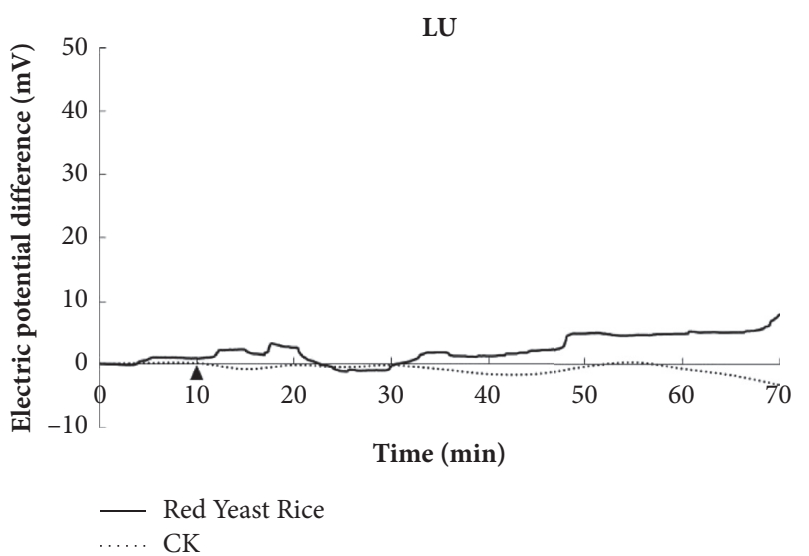

(a)

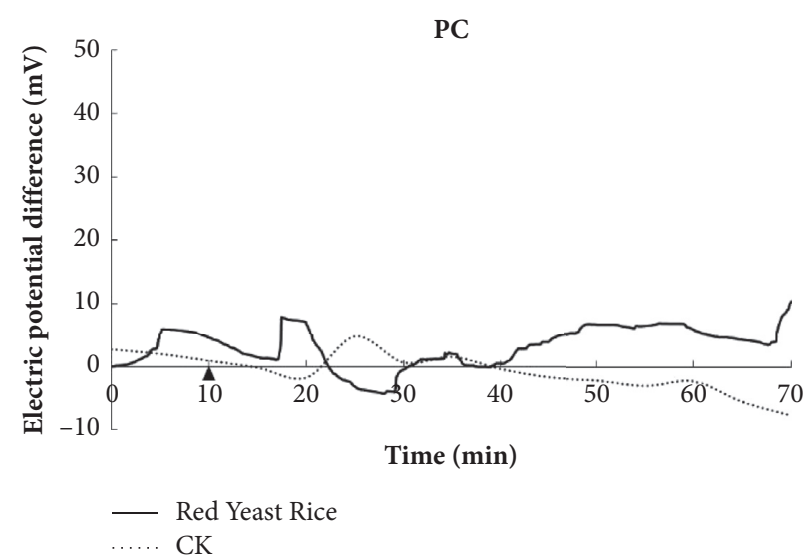

(b)

FIgURE 3: Electric potential difference of the lung meridian (a) and pericardium meridian (b).

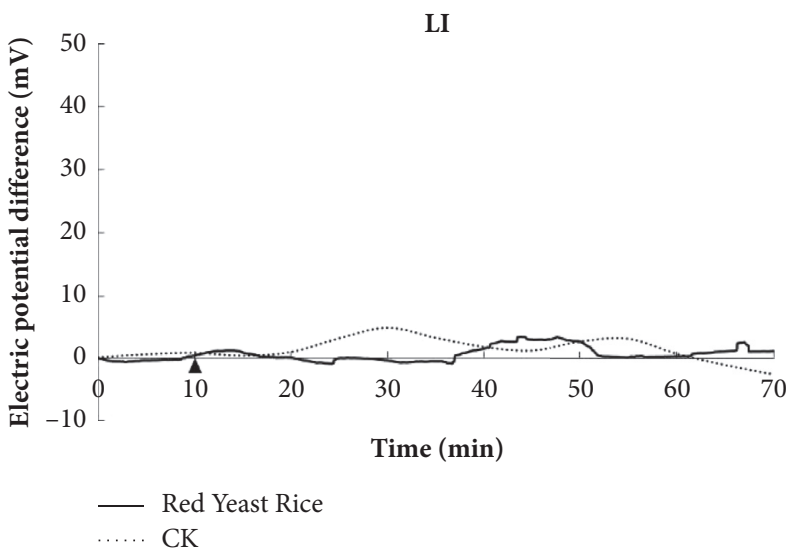

(a)

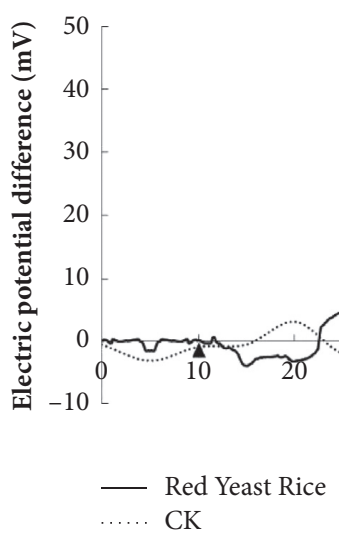

BL

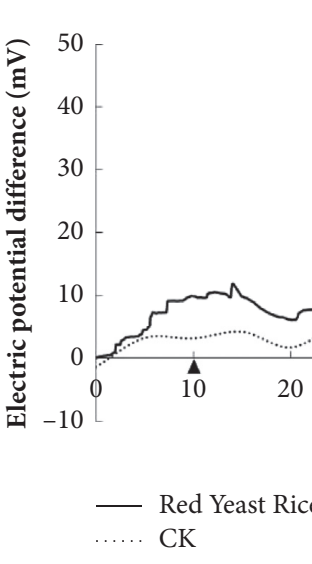

SP

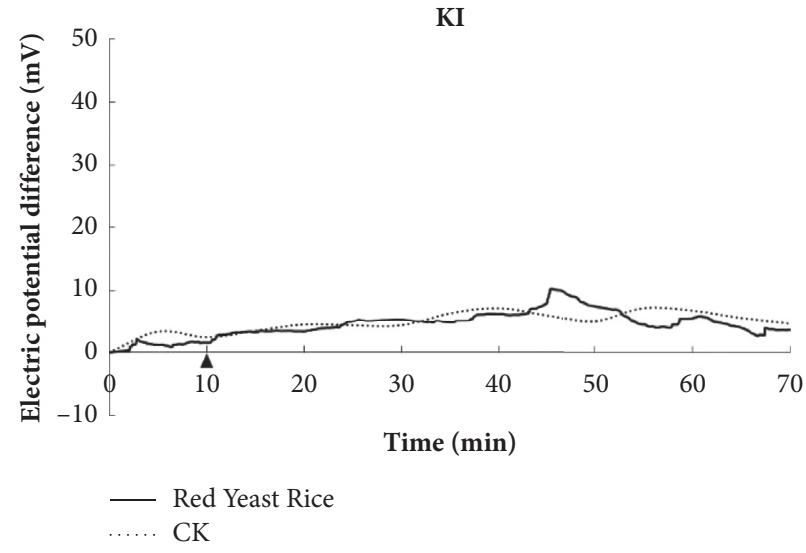

(d)

Figure 4: Continued. 


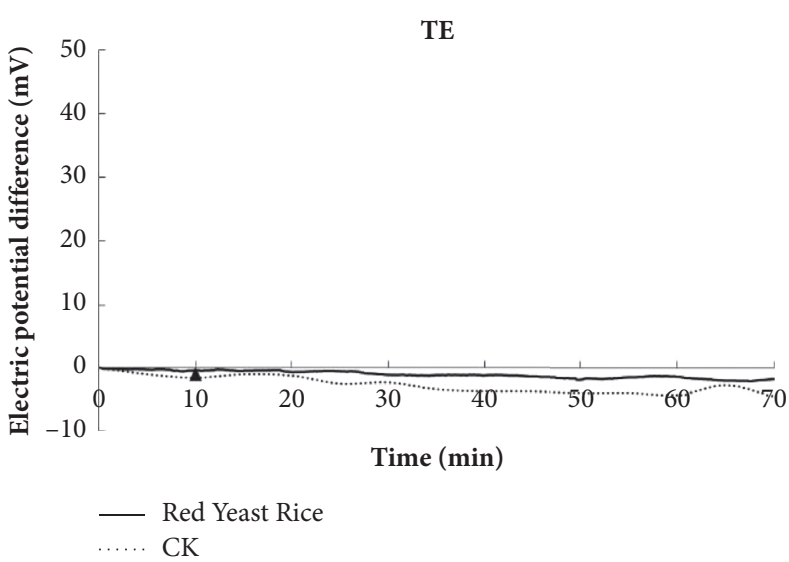

(e)

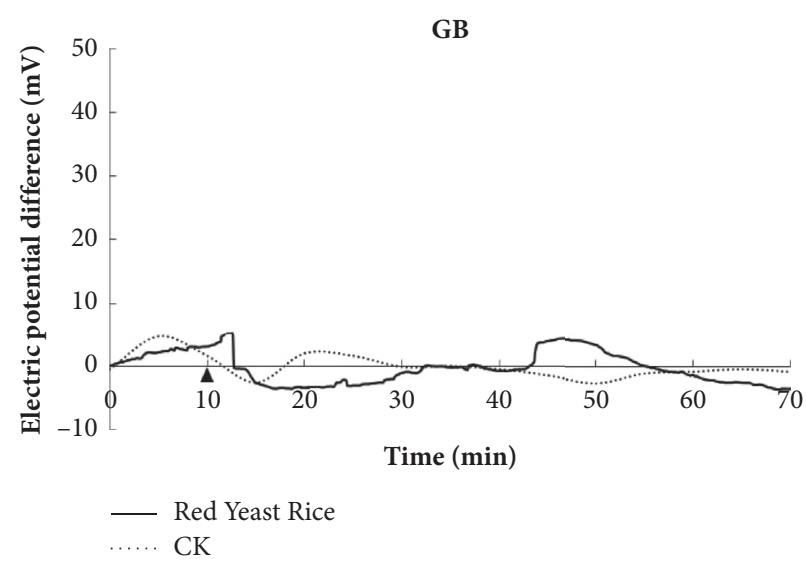

(f)

Figure 4: Electric potential difference of the large intestine meridian (a), spleen meridian (b), bladder meridian (c), kidney meridian (d), triple energizer meridian (e), and gallbladder meridian (f).

TABle 2: Antioxidant activity of red yeast rice and distilled water.

\begin{tabular}{lcc}
\hline Determination method & Red yeast rice & Distilled water \\
\hline PH & $4.22 \pm 0.19^{* *}$ & $7.01 \pm 0.01$ \\
Redox potential $(\mathrm{mV})$ & $359.63 \pm 6.68^{* *}$ & $512.70 \pm 9.36$ \\
ABTS (equal to the amount of Trolox standard solution substance) (mmol) & $0.48 \pm 0.11^{* *}$ & $0.02 \pm 0.00$ \\
FRAP (amount of $\mathrm{FeSO}_{4}$ standard solution substance) (mmol) & $0.080 \pm 0.004^{* *}$ & $0.01 \pm 0.00$ \\
T-SOD (U) & $4.71 \pm 0.19^{* *}$ & $0.09 \pm 0.00$ \\
\hline
\end{tabular}

Note: compared with distilled water, ${ }^{*} P<0.05,{ }^{* *} P<0.01 . n=5$.

content of some free radicals to give full play to their antioxidant effect, thus causing changes in the meridian EPD value [10-17]. Compared with other acupoints, the EPD signal-to-noise ratio between the 12 meridians on the left side of the Source-Sea (Yuan-He) acupoint is higher and suitable for monitoring [10]. The administration of red yeast rice can cause significant changes in the meridians of the stomach, heart, small intestine, and liver, mild and moderate in the lung meridian and pericardium meridian, but has no obvious effect on the others. Medical scientists in the past dynasties summarized the natural taste and meridian tropism of red yeast rice, concluding that it has sweet taste, smooth nature, and nontoxic. The meridian tropism of red yeast rice is the meridian of the large intestine, spleen, and liver [29]. Modern pharmacology has shown that red yeast rice exerts potential protective effects on the liver, pancreas, blood vessels, and intestines [2], which matches the functions to the meridians of the liver, stomach, heart, and small intestine, respectively. Therefore, the results of this study have reference significance for the treatment of diseases related to stomach, heart, and small intestine meridians by red yeast rice beyond the meridian of the large intestine, spleen, and liver [29].

The meridian orientation of traditional Chinese medicine refers to the selective effect of traditional Chinese medicine on different organs and the specificity of traditional Chinese medicine on meridians based on the theory of meridians [6-9]. The meridian tropism has been proved to be effective in guiding clinical practice, but other than the scientifically mysterious bioenergy or Qi concept, there is hardly any clue to what exactly happens immediately after a medication and could be observed precisely. It is still difficult to determine how red yeast rice selectively influences the electrical properties of particular meridians; however, this study attempts to explore the physiological effects of red yeast rice on the human body by monitoring the meridian EPD, so as to provide new ideas for the analysis of the specificity of red yeast rice on the meridian, the application, and development of red yeast rice.

\section{Conclusions}

In this study, the $\mathrm{pH}$ value of the red yeast rice was 4.22 , the ORP was $359.63 \mathrm{mV}$, the ABTS was $0.48 \mathrm{mmol}$ Trolox, the FRAP was $0.08 \mathrm{mmol} \mathrm{FeSO}_{4}$, the T-SOD was $4.71 \mathrm{U}$, and the average particle size was $108 \mathrm{~nm}(7.1 \%)$ and $398.1 \mathrm{~nm}$ (92.9\%). The results of 12 acupuncture meridians EPD showed that the red yeast rice can significantly affect the EPD of stomach, heart, small intestine, and liver meridians.

\section{Data Availability}

The data that support the findings of this study are openly available in Mendeley Data at https://dxdoi.org/10.17632/ txc5g64t6s.1.

\section{Conflicts of Interest}

The authors declare no conflicts of interest regarding the publication of this paper. 


\section{Acknowledgments}

This research was supported by the National Natural Science Foundation of China (31500685); Zhejiang Provincial Natural Science Foundation of China (LY16C050002); Zhejiang University Rockchip United Technologies Center Foundation (ZD20190052); Education Research Project for Young and Middle-Aged Teachers in Fujian Province of China (JAT200947); and Fujian Provincial Innovative Training Programme for College Students (S202013470027 and S202013470029).

\section{References}

[1] J. Song, J. Luo, Z. Ma et al., "Quality and authenticity control of functional red yeast rice-a review," Molecules, vol. 24, no. 10, p. 1944, 2019.

[2] J. Hu, J. Wang, Q.-X. Gan et al., "Impact of red yeast rice on metabolic diseases: a review of possible mechanisms of action," Journal of Agricultural and Food Chemistry, vol. 68, no. 39, pp. 10441-10455, 2020.

[3] Z. Xiong, X. Cao, Q. Wen et al., "An overview of the bioactivity of monacolin K/lovastatin," Food and Chemical Toxicology, vol. 131, Article ID 110585, 2019.

[4] L. R. Gomes and P. Leão, "Recent approaches on signal transduction and transmission in acupuncture: a biophysical overview for medical sciences," Journal of Acupuncture and Meridian Studies, vol. 13, no. 1, pp. 1-11, 2020.

[5] H. Zhang, G. Han, and G. Litscher, "Traditional acupuncture meets modern nanotechnology: opportunities and perspectives," Evidence-Based Complementary and Alternative Medicine, vol. 2019, Article ID 2146167, 2019.

[6] P. Liu, S. Liu, G. Chen, and P. Wang, "Understanding channel tropism in traditional Chinese medicine in the context of systems biology," Frontiers of Medicine, vol. 7, no. 3, pp. 277-279, 2013.

[7] M. Zeng, M. Li, L. Zhang et al., "Different meridian tropism in three Chinese medicines: Tinglizi (Semen Lepidii Apetali), Yiyiren (Semen Coicis), Cheqianzi (Semen Plantaginis)," Journal of traditional Chinese medicine = Chung I Tsa Chih Ying Wen Pan, vol. 39, no. 2, pp. 213-220, 2019.

[8] L.-L. Wang, W.-W. Li, C.-S. Wu et al., "Relationship between tissue distributions of modified Wuzi Yanzong prescription (加味五子衍宗方) in rats and meridian tropism theory," Chinese Journal of Integrative Medicine, vol. 24, no. 2, pp. 117-124, 2018.

[9] N. Zhang, Y. Hua, C. Wang et al., "Distribution study of tryptanthrin in rat tissues by HPLC and its relationship with meridian tropism of indigo naturalis in traditional Chinese medicine," Biomedical Chromatography, vol. 28, no. 12, pp. 1701-1706, 2014.

[10] J.-K. Guo, J.-S. Xu, T.-B. Chen et al., "Effects of TAT-SOD at acupoints on essential hypertension by monitoring meridians electrical potential," Chinese Journal of Integrative Medicine, vol. 26, no. 9, pp. 694-700, 2020.

[11] M. M. Xu, J. K. Guo, J. S. Xu et al., "Monitoring the effects of acupoint antioxidant intervention by measuring electrical potential difference along the meridian," Evidence-Based Complementary and Alternative Medicine, vol. 2015, Article ID 286989, 2015.

[12] P. Rao, H. Chen, H. Zhou et al., "Studies on anti-oxidative capacity, thermal stability and its channel tropism of cordyceps militaris," Journal of Chinese Institute of Food Science and Technology, vol. 20, no. 7, pp. 59-64, 2020.

[13] P. Rao, Q. Chen, H. Zhou et al., "Preliminary study on the antioxidant capacity of water extracts derived from Inonotus obliquus (Fr.) Pilat and its effect on human meridian voltage," Journal of Chinese Institute of Food Science and Technology, vol. 20, no. 6, pp. 88-94, 2020.

[14] S. Yan, J. Guo, M. Xu et al., "Study on the antioxidant activity of Dendrobium officinale Kimura et Migo and its different effects on Zang-Fu organs channel tropism," Chinese Journal of Integrated Traditional and Western Medicine, vol. 41, no. 1, pp. 41-45, 2021.

[15] W. Lu, J. Guo, J. Zhou et al., "Hypothesis review: the direct interaction of food nanoparticles with the lymphatic system," Food Science and Human Wellness, vol. 1, no. 1, pp. 61-64, 2012.

[16] M. Xu, J. Guo, L. Ni et al., "The effect of infusions of tea soup on electrical potential difference along the meridian," Journal of Chinese Institute of Food Science and Technology, vol. 18, no. 11, pp. 27-33, 2018.

[17] Q. Chen, J. Guo, M. Xu et al., "Anti-oxidative analysis of lapsang tea infusion, tea polyphenols, tea polysaccharides and its electrophysiological acupuncture meridian signals," Journal of Chinese Institute of Food Science and Technology, vol. 20, no. 10, pp. 43-49, 2020.

[18] World Health Organization Regional Office for the Western Pacific, WHO Standard Acupuncture Point Locations in the Western Pacific Region, World Health Organization, Geneva, Switzerland, 2008.

[19] Ż. Król-Kilińska, D. Kulig, I. Yelkin, A. Zimoch-Korzycka, Ł. Bobak, and A. Jarmoluk, "The effect of using micro-clustered water as a polymer medium," International Journal of Molecular Sciences, vol. 22, no. 9, p. 4730, 2021.

[20] J. Guo, S. Liu, X. Cheng et al., "Revealing acupuncture meridian-like system by reactive oxygen species visualization," Bioscience Hypotheses, vol. 2, no. 6, pp. 443-445, 2009.

[21] J. Guo, H. Xu, S. Liu et al., "Visualising reactive oxygen species in live mammals and revealing of ROS-related system," Free Radical Research, vol. 53, no. 11-12, pp. 1073-1083, 2019.

[22] J. Guo, Y. Chen, B. Yuan, S. Liu, and P. Rao, "Effects of intracellular superoxide removal at acupoints with TAT-SOD on obesity," Free Radical Biology and Medicine, vol. 51, no. 12, pp. 2185-2189, 2011.

[23] J. K. Guo, M. M. Xu, M. F. Zheng et al., "Topical application of TAT-superoxide dismutase in acupoints LI 20 on allergic rhinitis," Evidence-Based Complementary and Alternative Medicine, vol. 2016, Article ID 28119757, 2016.

[24] D. Lin, W. Lin, G. Gao et al., "Purification and characterization of the major protein isolated from Semen Armeniacae Amarum and the properties of its thermally induced nanoparticles," International Journal of Biological Macromolecules, vol. 159, pp. 850-858, 2020.

[25] L. Cai, C. Lin, N. Yang et al., "Preparation and characterization of nanoparticles made from co-incubation of SOD and glucose," Nanomaterials (Basel), vol. 7, no. 12, p. 458, 2017.

[26] J. Zhou, J. Liu, D. Lin et al., "Boiling-induced nanoparticles and their constitutive proteins from Isatis indigotica Fort. root decoction: purification and identification," Journal of Traditional and Complementary Medicine, vol. 7, no. 2, pp. 178187, 2017.

[27] L. Ke, H. Wang, G. Gao et al., "Direct interaction of food derived colloidal micro/nano-particles with oral macrophages," NPJ Science of Food, vol. 1, p. 3, 2017. 
[28] L. J. Ke, G. Z. Gao, Y. Shen, J. W. Zhou, and P. F. Rao, "Encapsulation of aconitine in self-assembled licorice protein nanoparticles reduces the toxicity in vivo," Nanoscale Research Letters, vol. 10, no. 1, p. 449, 2015.

[29] B. Zhu, F. Qi, J. Wu et al., "Red yeast rice: a systematic review of the traditional uses, chemistry, pharmacology, and quality control of an important Chinese folk medicine," Frontiers in Pharmacology, vol. 10, p. 1449, 2019. 